\title{
Suppression of experimental
} autoimmune encephalomyelitis by ultraviolet light is not mediated by isomerization of urocanic acid

\author{
Amy A. Irving, Steven J. Marling, Lori A. Plum and Hector F. DeLuca*
}

\begin{abstract}
Background: Ultraviolet B irradiation confers strong resistance against experimental autoimmune encephalomyelitis, a model of multiple sclerosis. This protection by ultraviolet $B$ is independent of vitamin $D$ production but causes isomerization of urocanic acid, a naturally occurring immunosuppressant.

Methods: To determine whether UCA isomerization from trans to cis is responsible for the protection against experimental autoimmune encephalomyelitis afforded by ultraviolet B, trans- or cis-urocanic acid was administered to animals and their disease progression was monitored.

Results: Disease incidence was reduced by $74 \%$ in animals exposed to ultraviolet B, and skin cis-urocanic acid levels increased greater than 30\%. However, increasing skin cis-urocanic acid levels independent of ultraviolet B was unable to alter disease onset or progression.

Conclusions: It is unlikely that urocanic acid isomerization is responsible for the ultraviolet B-mediated suppression of experimental autoimmune encephalomyelitis. Additional work is needed to investigate alternative mechanisms by which UVB suppresses disease.
\end{abstract}

\section{Background}

Multiple sclerosis (MS), a demyelinating disease of the central nervous system, is less prevalent in locations that receive greater amounts of sunlight. This observation has led to the hypothesis that vitamin D might play a major role in preventing the disease. However, despite a correlation between low serum 25-hydroxyvitamin D (25-OH-D) levels and higher disease incidence in human populations, evidence that vitamin $\mathrm{D}$ can reduce the incidence of MS has so far not been obtained [1-3]. In experimental autoimmune encephalomyelitis (EAE), a model for MS, 1,25-dihydroxyvitamin $\mathrm{D}_{3}\left(1,25-(\mathrm{OH})_{2} \mathrm{D}_{3}\right)$ does reduce disease, but only when accompanied by elevated serum calcium $[4,5]$. Strikingly, mice deficient in vitamin

*Correspondence: deluca@biochem.wisc.edu

Department of Biochemistry, University of Wisconsin-Madison, 433 Babcock Drive, Madison, WI 53706, USA
$\mathrm{D}$ or lacking the vitamin $\mathrm{D}$ receptor have each been shown to be resistant to developing EAE [6, 7].

MS is an autoimmune disease in which activated T cells target and cause the destruction of the myelin sheath in the central nervous system. In addition to its role in generating vitamin $\mathrm{D}$ in the skin, ultraviolet light can cause systemic suppression of the immune system [8]. Ultraviolet $B$ (UVB) irradiation, especially the narrow band from 300 to $315 \mathrm{~nm}$, confers resistance against EAE [9]. This narrow band is exclusive of that required to generate vitamin D in the skin. UV light must then confer protection by mechanisms distinct from vitamin $\mathrm{D}$.

One candidate for this protection is the chromophore urocanic acid (UCA), an intermediate in the catabolism of L-histidine [10]. Under normal conditions, urocanic acid accounts for up to $0.5 \%$ of the dry weight of the epidermis, the majority of which is the trans isomer [11]. Upon UV exposure of the epidermis, trans-UCA 
isomerizes to the cis isomer, which possesses immunosuppressive properties. The greatest degree of isomerization occurs within the same window demonstrated to offer protection from EAE (300-310 nm) [12]. Further, MS patients have significantly lower blood levels of cisUCA compared to controls [13].

In the current study, we tested trans- and cis-UCA, as well as trans-UCA plus UVB for potential to prevent the onset or progression of EAE.

\section{Methods}

\section{Animals and diet}

Female C57BL/6 mice were purchased from Jackson Laboratory at 8-9 weeks of age and allowed to acclimate to the facility for $\sim 1$ week before experimental manipulations began. Mice were maintained in the Department of Biochemistry vivarium with a $12 \mathrm{~h}: 12 \mathrm{~h}$ light:dark cycle and fed a standard lab diet chow 5008 (Purina Mills, Richmond, IN). All procedures were approved by the Research Animal Resources Committee of the College of Agricultural and Life Sciences at the University of Wisconsin-Madison.

\section{EAE induction and scoring}

Mice were immunized at 9-10 weeks of age with myelin oligodendrocyte glycoprotein peptide (MOG) ${ }_{35-55}$ purchased from Hooke Laboratories (Lawrence, MA). MOG $_{35-55}$ (MEVGWYRSPFSRVVHLYRNGK) is emulsified in complete Freund's adjuvant and contains inactivated Mycobacterium tuberculosis H37Ra. On day 1, each mouse was injected subcutaneously with $10 \mu \mathrm{l}$ of MOG emulsion and injected intraperitoneally with $200 \mathrm{ng}$ of pertussis toxin (List Biological Laboratories, Campbell, CA) diluted in sterile PBS. On day 3, a second $200 \mathrm{ng}$ booster pertussis toxin injection was given (Fig. 1). Each mouse was scored daily Monday through Friday for clinical signs of EAE using the following scale: 0 , no clinical disease; 1 , loss of tail tone; 2 , unsteady gait; 3 , hind limb paralysis; 4 , forelimb paralysis; 5 , death.

\section{UVB radiation treatment}

The dorsal surface of each mouse was shaven using an electric razor prior to treatment. Mice were irradiated with a bank of UVB lamps that emit from 300 to $315 \mathrm{~nm}$ with a peak at $311 \mathrm{~nm}$ (Amjo Corp, West Chester, $\mathrm{OH}$ ). The radiation output was measured by placing a UV radiometer equipped with 302 and $365 \mathrm{~nm}$ sensors (UVP LLC, Upland, CA) at five locations within the cage, representing the positions occupied by the mice. An average output was calculated and the time adjusted to expose the mice to $10 \mathrm{~kJ} / \mathrm{m}^{2}$ per treatment. These readings were confirmed using a wide band spectroradiometer RPS900 (International Light, Peabody, MA). Mice were irradiated daily Monday through Friday for four weeks (Fig. 1).

\section{Chemicals}

Cis-urocanic acid (U6883) was purchased from SigmaAldrich (St. Louis, MO) and trans-urocanic acid (I0002) was purchased from TCI America (Portland, OR). Both compounds had been determined by the manufacturer to be greater than $98 \%$ pure by HPLC.

For topical dosing studies, the compounds were prepared by diluting commercially available trans- or cis-UCA at a concentration of $1 \mathrm{mg} / \mathrm{ml}$ in ethanol (to facilitate rapid skin absorption). Each mouse was then given $2 \times 0.2 \mathrm{ml}(400 \mu \mathrm{g}$ total) of either trans- or cis-UCA onto shaved dorsal skin on Monday, Wednesday and Friday each week during the study (Fig. 1). For the study involving dosing with trans-UCA followed by exposure of the mouse to UV, dosing and exposure occurred daily Monday through Friday each week (Fig. 1). Formulations were prepared weekly and concentrations confirmed by HPLC. UCA diluted in ethanol and stored at $4{ }^{\circ} \mathrm{C}$ is stable for at least 3 months.

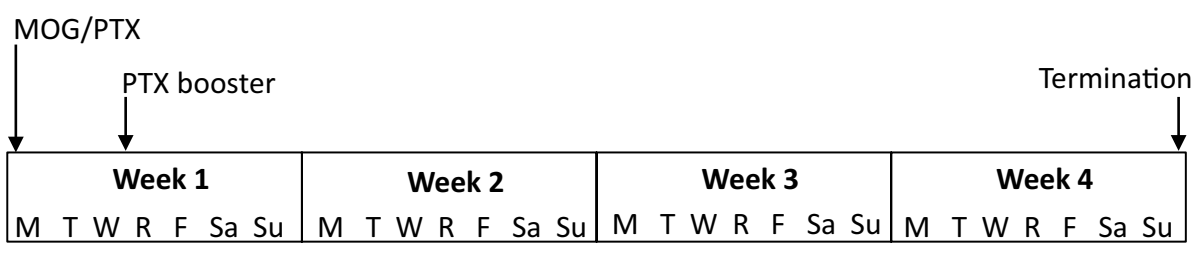

Figs $2 \& 4$ $\bullet \bullet \bullet \bullet \bullet$

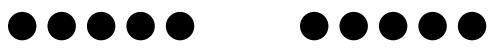

Figs $3 \& 6$

Fig. 1 Schematic of the experimental timeline for each experiment. The days of the week are denoted by abbreviations below each of the week designations. The immunization schedule and termination are shown above the timeline. Each closed circle represents an experimental exposure (to either UVB, compound or the combination). The Figures listed to the left of the circles are those which correspond to that particular experimental timeline. MOG myelin oligodendrocyte glycoprotein; PTX pertussis toxin 
For the i.p. dosing study, trans-UCA was prepared as above but diluted in sterile PBS. Mixed UCA isomers were generated by exposing $1 \mathrm{mg} / \mathrm{ml}$ trans-UCA to narrow band UVB (300-315 $\mathrm{nm}$ ) for $30 \mathrm{~min}(13.5 \mathrm{~kJ})$. The percent conversion to cis-UCA was then measured by HPLC and the absolute concentration of trans- and cis-UCA determined using quantitative standards. Each mouse was given $0.2 \mathrm{ml}(200 \mu \mathrm{g})$ of either transUCA or mixed UCA isomers via i.p. injection on Monday, Wednesday and Friday each week during the study (Fig. 1). Formulations were prepared weekly and concentrations confirmed by HPLC. UCA diluted in PBS and stored at $4{ }^{\circ} \mathrm{C}$ is stable for at least 6 months.

\section{Sample preparation}

At termination, mice were euthanized with $\mathrm{CO}_{2}$ and skin was collected from the dorsal surface, cut into small pieces and frozen at $-80{ }^{\circ} \mathrm{C}$. A modified Bligh-Dyer method was used to isolate water soluble components, which includes UCA. Skin samples were homogenized on ice in cold water, followed by the addition of methanol/ dichloromethane, dichloromethane, and finally water. Samples were then centrifuged for $15 \mathrm{~min}$ at $3500 \mathrm{rpm}$ at $4{ }^{\circ} \mathrm{C}$. After centrifugation, the water phase was collected, filtered first through a $0.45 \mu \mathrm{m}$ filter and then again through a $0.22 \mu \mathrm{m}$ filter, dried down under nitrogen and resuspended in HPLC mobile phase (water containing $0.1 \%$ trifluoroacetic acid (TFA)).

\section{HPLC methods}

HPLC separation was performed on a Waters system, composed of a 717plus Autosampler, 600 Controller and Pump, and 996 Photodiode Array Detector (PDA). Empower Pro V5.0 software was used for data acquisition and to control the HPLC system. A Waters Symmetry C18 column $(3.9 \times 150 \mathrm{~mm}, 5 \mu \mathrm{m})$ maintained at $30{ }^{\circ} \mathrm{C}$ was used for separation. A gradient from $100 \%$ water to 70:30 water:ACN was run over a $15 \mathrm{~min}$ period at a flow rate of $0.5 \mathrm{~mL} / \mathrm{min}$. Mobile phase contained $0.1 \%$ TFA. Data were collected at $264 \mathrm{~nm}$. Retention time for transUCA was $10.3 \mathrm{~min}$ and cis-UCA was $14.2 \mathrm{~min}$.

\section{Statistical analysis}

Data are expressed as Mean $\pm \mathrm{SD}$. Onset was calculated by averaging the first day when clinical signs appeared. Unless otherwise noted, average clinical scores were calculated at termination on day 28. Mean severity was determined by averaging all clinical scores within a treatment group for a particular day. Statistical calculations indicate that 8 mice per group are required to detect an $80 \%$ reduction in average clinical score with $90 \%$ power at a significance level of 0.05 , as is seen with UVB exposure. If any urocanic acid intervention showed half the efficacy as UVB exposure ( $40 \%$ reduction in average clinical score), 9 mice per group would give $90 \%$ power at a significance level of 0.05 . Based on these calculations we have employed 10-12 mice per group to ensure $>90 \%$ power to detect a statistical difference, if one exists.

Statistical analysis was performed using the two-tailed Fisher exact probability test for incidence, the Wilcoxon rank sum test for clinical scores, and the Kendall rank test for correlation between disease indices and UCA content. A value of $\mathrm{P}<0.05$ was considered statistically significant.

\section{Results \\ Conversion of urocanic acid by narrow band UVB}

Narrow band (NB) UVB was efficient at converting trans-UCA to the cis isomer both in vitro in solution and in vivo in skin. In vitro, starting material was confirmed to be greater than $98 \%$ pure trans isomer by HPLC. Approximately $13.5 \mathrm{~kJ}$ of NB UVB was needed to convert half of the trans to the cis isomer.

In vivo, under normal conditions, less than $1 \%$ of skin UCA content was in the cis form. After 4 weeks of UVB exposure, as much as $35 \%$ of total skin UCA was the cis isomer (Fig. 2a). UVB treatment resulted in a significant suppression of EAE (Fig. 2b). While 100\% (24/24) of animals immunized with MOG developed EAE with an average onset of $13.9 \pm 3.5$ days, UVB reduced incidence to $26 \%(6 / 23, \mathrm{P}<0.0001)$ and delayed onset to $20.2 \pm 4.6$ days $(\mathrm{P}=0.006)$. UVB also reduced mean clinical score at study termination to $0.8 \pm 1.4$, compared to $3.8 \pm 0.7$ in control animals $(\mathrm{P}<0.00001)$. While UVB exposure tripled the total UCA skin content (Fig. 2c), there was no correlation between total or isoform-specific UCA content and day of onset or terminal disease score (data not shown).

\section{Topical urocanic acid does not prevent EAE}

Topical trans- or cis-UCA was applied daily to shaven dorsal skin of EAE-induced mice. Indicative of its immunosuppressive properties, topical cis decreased average spleen weight $30 \%$ compared to vehicle treated animals $(\mathrm{P}<0.008$, Fig. 3a). Despite this, neither isomer protected against EAE development (Fig. 3b), with an incidence of $100 \%$ in each of the groups. Mean onset did not differ from vehicle $(17.6 \pm 4.9$ days $)$ for animals given either trans $(16.5 \pm 4.6$ days, $\mathrm{P}=0.4)$ or cis $(14.4 \pm 2.9$ days, $\mathrm{P}=0.1$ ) compound. Mean clinical score at study termination also did not differ significantly between groups: vehicle $3.3 \pm 1.1$, trans $3.1 \pm 1.0(\mathrm{P}=1$ versus vehicle), cis $4.1 \pm 0.9(\mathrm{P}=0.07$ versus vehicle $)$.

A second set of experiments was conducted to determine whether increasing skin trans content topically and then exposing that skin to UVB could increase 
a UCA Isomer Composition

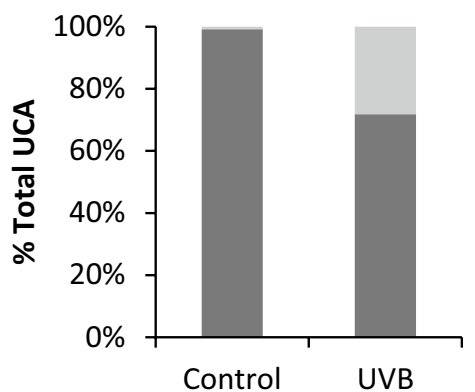

b

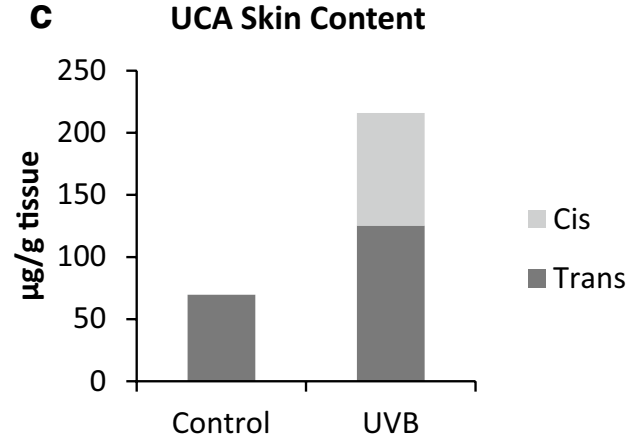

EAE Disease Index

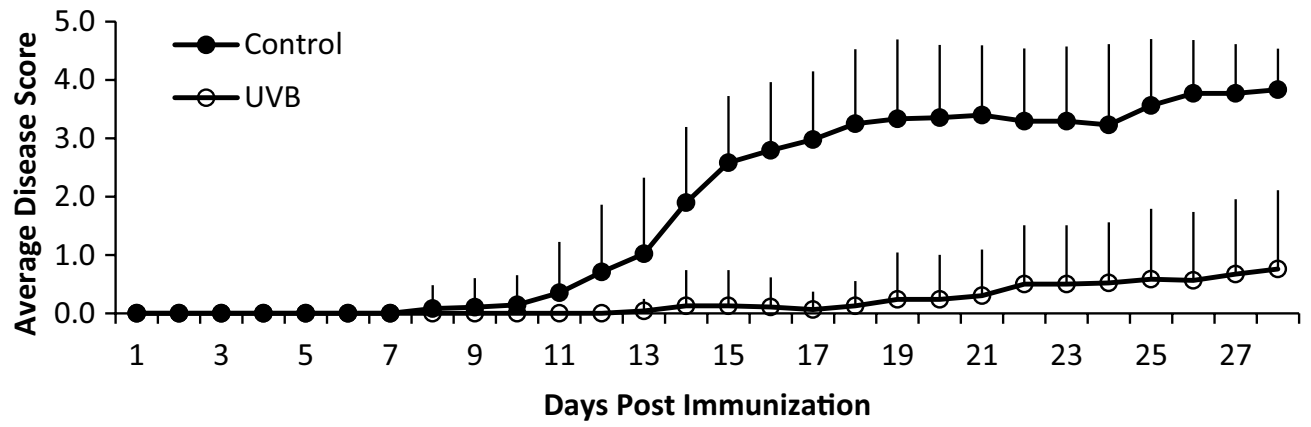

\begin{tabular}{|c|c|c|c|}
\hline Group & Incidence (n, \%) & Day of Onset & Average Clinical Score \\
\hline Control & $24 / 24,100 \%$ & $13.9 \pm 3.5$ & $3.8 \pm 0.7$ \\
\hline UVB & $6 / 23(26 \%)$ & $20.2 \pm 4.6$ & $0.8 \pm 1.4$ \\
\hline
\end{tabular}

Fig. 2 UVB increases cis-UCA skin content and efficiently suppresses EAE. a UVB significantly increased skin cis-UCA content $(P<0.0001)$ through conversion of the trans isomer $(\mathbf{b})$. $\mathbf{c} \cup V B$ suppresses the onset $(P=0.006)$ and progression of $E A E(P<0.0001)$. Data are cumulative of two independent experiments

conversion to the cis isoform and offer the same or greater protection than UVB alone. While topical application of trans-UCA did increase skin trans content $(P=0.03$, Fig. 4a), spleen weight was unaffected $(P=0.2$, Fig. 4b) and disease incidence (12/12) did not differ from vehicle $(23 / 24, P=1)$. The greatest disease suppression occurred with UVB exposure alone, where spleen weight was significantly increased $(\mathrm{P}<0.03)$ and incidence significantly decreased $(10 / 20, \mathrm{P}<0.001)$ compared to vehicle (Fig. 4c). When animals given the trans isomer were then exposed to UVB, spleen weight was unaffected compared to vehicle $(\mathrm{P}=0.2$, Fig. $4 \mathrm{~b})$ with an incidence of $65 \%(15 / 23, P=0.01$ versus vehicle, $P=0.4$ versus $U V B$ alone) (Fig. 4c).

Interestingly, animals given supplemental trans topically and then exposed to UVB were able to convert to the cis isoform with similar efficiency to that of animals only exposed to UVB with no additional UCA. However, UVB alone was the only treatment able to significantly delay onset of disease (21.4 \pm 6.6 days) compared to vehicle $(16.2 \pm 3.0$ days, $\mathrm{P}<0.03)$. Mean clinical score at study termination only differed from vehicle $(2.2 \pm 1.0)$ for animals in the UVB group $(0.9 \pm 1.2, \mathrm{P}<0.0008)$, and did not differ for either the trans $(2.0 \pm 1.1, \mathrm{P}=0.8)$ or trans plus UVB groups $(1.4 \pm 1.4, \mathrm{P}=0.2)$. Further, there was no correlation between terminal disease score or day of onset and total skin UCA content or \% total skin UCA in the cis form (Fig. 5), nor absolute trans or cis skin UCA content (data not shown).

\section{Systemic delivery of urocanic acid isomers does not prevent EAE}

To test whether systemic delivery of a mixture of UCA isomers similar to that produced in skin upon UVB exposure could protect against EAE development, mice were injected i.p. three times per week for four weeks with $200 \mu \mathrm{g}$ of either trans-UCA or mixed UCA isomers containing $40 \%$ trans and $60 \%$ cis (generated by 
a Spleen Weight

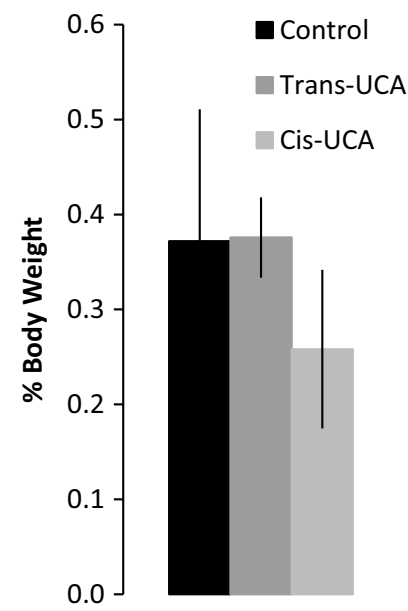

b

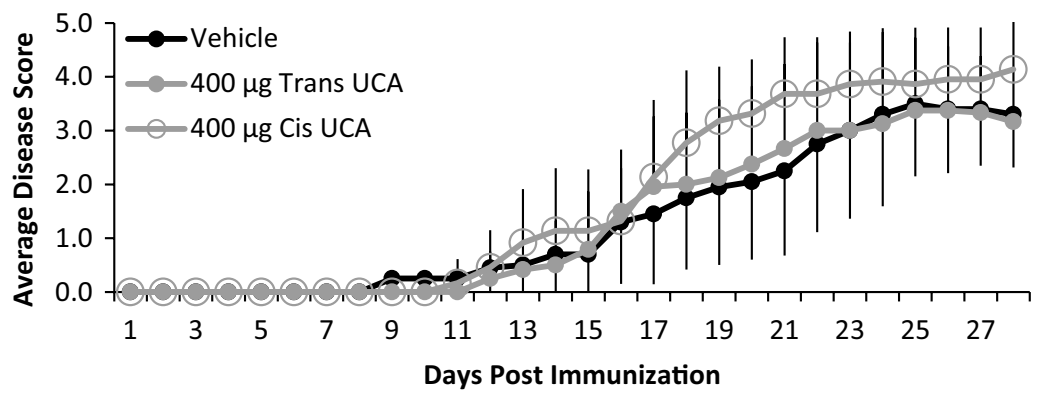

\begin{tabular}{|c|c|c|c|}
\hline Group & Incidence $(\mathbf{n}, \%)$ & Day of Onset & Average Clinical Score \\
\hline Vehicle & $12 / 12(100 \%)$ & $17.6 \pm 4.9$ & $3.3 \pm 1.1$ \\
\hline $400 \mu \mathrm{g}$ Trans UCA & $12 / 12(100 \%)$ & $16.5 \pm 4.6$ & $3.1 \pm 1.0$ \\
\hline $400 \mu \mathrm{g}$ Cis UCA & $12 / 12(100 \%)$ & $14.4 \pm 2.9$ & $4.1 \pm 0.9$ \\
\hline
\end{tabular}

Fig. 3 Topical UCA is unable to suppress EAE. a Cis-UCA significantly decreased the spleen:body weight ratio, while trans-UCA had no effect. Error bars are SD from the Mean and represent biological variation. b Neither UCA isomer was able to suppress EAE onset or progression. Data are representative of one experiment

exposing a solution of trans-UCA to UVB). Neither compound improved disease outcome compared to vehicle (Fig. 6). Incidence was high in all groups: $100 \%$ $(10 / 10)$ for vehicle, $70 \%(7 / 10)$ for trans $(\mathrm{P}=0.2$ versus vehicle), and $100 \%(10 / 10)$ for the mixed UCA isomers. Mean day of onset did not differ significantly between groups: $16.2 \pm 3.0$ days for vehicle, $16.1 \pm 5.8$ days for trans ( $\mathrm{P}=0.3$ versus vehicle), and $13.6 \pm 2.8$ days for mixed UCA isomers $(\mathrm{P}=0.06$ versus vehicle). There was also no difference in mean disease score at termination from control $(2.7 \pm 0.9)$ for either the trans $(1.9 \pm 2.0, \mathrm{P}=0.4)$ or mixed UCA isomer $(2.5 \pm 1.2$, $\mathrm{P}=0.6)$ groups.

\section{Discussion}

MS is not only more prevalent with increasing distance from the equator, but deaths from the disease [14], as well as relapse [15], are also more common. This led to the hypothesis that the vitamin D produced by UVB acting on 7-dihydrocholesterol was responsible for the lowered incidence of MS. However, our group has shown that the vitamin D receptor (VDR) is required for EAE initiation [6], and several groups have demonstrated that animals deficient for vitamin D are protected against EAE $[6,7$, 16]. Further, administration of the active form of vitamin D, i.e. $1,25-(\mathrm{OH})_{2} \mathrm{D}_{3}$, can suppress EAE in mice but only when hypercalcemia results [5]. Hypercalcemia produced by parathyroid hormone also suppresses EAE [4]. Thus, the idea that vitamin D mediates the suppression of MS by UVB is no longer viable.
As an alternative hypothesis, isomerization of urocanic acid was an attractive candidate to explain the suppression by UVB. Peak isomerization occurs in the UV range most able to suppress EAE [12], the immunosuppressive properties of the cis isomer has been demonstrated in multiple animal models [17-19], and an association between low cis-UCA levels and MS in patients has been shown [13]. However, we have demonstrated that increasing skin cis-UCA levels independent of UVB exposure is unable to protect against EAE.

Many previous studies have examined the effects of a single dose of UCA on various disease outcomes. However, a single UV exposure can increase skin levels of UCA in humans for weeks [20]. Here, we demonstrate neither prolonged dermal nor transient systemic increases in cis-UCA are able to protect against EAE.

To increase cis content via isomerization, we administered the trans isomer topically prior to UVB exposure. However, the conversion to the cis isomer was not any greater than in animals exposed to UVB alone. This is likely owing to the achievement of the photostationary state where no further conversion to cis will occur [21]. Despite reaching this level, protection was actually dampened when UVB was accompanied by topical application of the trans isomer.

Cis-UCA levels in blood are low, and we were unable to detect significant differences in this measure between control and UVB-exposed mice. A single i.p. injection of UVB-exposed UCA caused a rapid rise in blood cis-UCA levels that returned to baseline within $4 \mathrm{~h}$. These low and 

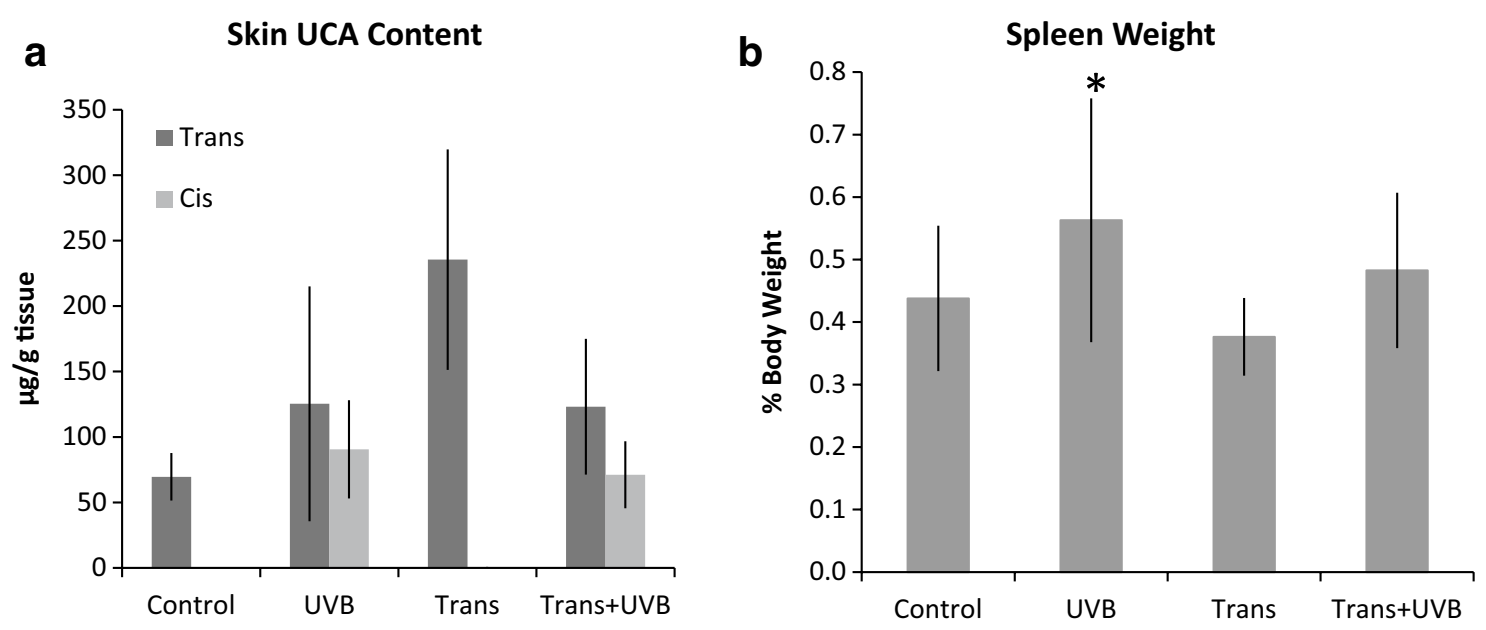

EAE Disease Index

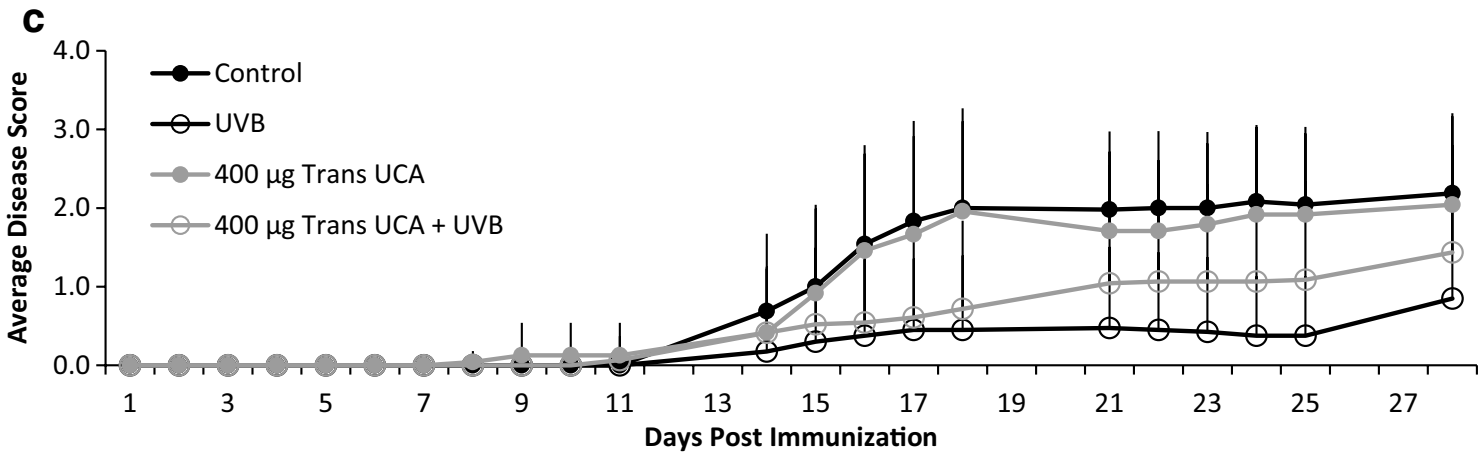

\begin{tabular}{|c|c|c|c|}
\hline Group & Incidence $(\mathbf{n}, \boldsymbol{\%})$ & Day of Onset & Average Clinical Score \\
\hline Control & $23 / 24(96 \%)$ & $16.2 \pm 3.0$ & $2.2 \pm 1.0$ \\
\hline UVB & $10 / 20(50 \%)$ & $21.4 \pm 6.6$ & $0.9 \pm 1.2$ \\
\hline $400 \mu g$ Trans UCA & $12 / 12(100 \%)$ & $16.6 \pm 3.0$ & $2.0 \pm 1.1$ \\
\hline $400 \mu g$ Trans UCA + UVB & $15 / 23(65 \%)$ & $19.0 \pm 5.3$ & $1.4 \pm 1.4$ \\
\hline
\end{tabular}

Fig. 4 Trans-UCA applied topically in combination with UVB is not as effective as UVB alone to suppress EAE. a Topical trans-UCA significantly increases skin trans content. Despite this, when combined with UVB, supplemental trans is converted to cis to levels similar to that in animals exposed to UVB alone. $\mathbf{b} U V B$ alone is the only group in which spleen:body weight ratio was significantly increased $(P<0.03)$. Error bars are SD from the Mean and represent biological variation. c Trans-UCA alone does not affect disease course. UVB greatly suppresses EAE onset and progression. When supplemental trans-UCA is given topically prior to UVB exposure, the disease suppression is dampened. Data are cumulative of two independent experiments

transient blood levels are likely explained by the presence of urocanase in the liver, which specifically metabolizes cis-UCA, and the high water solubility of the compound, causing it to be readily excreted.

It is clear that ultraviolet $\mathrm{B}$ exposure efficiently prevents $E A E$, though we find no evidence to support UCA isomerization as the mediator of this suppression. This is not the first study to discount UCA as a mediator of UV immunosuppression, or that UCA administration does not mimic the effects of UV exposure [17]. This lack of protection may not be surprising. Recent evidence has emerged demonstrating the essential role for regulatory $B$ cells in the protection by UVB from EAE [22]. By contrast, urocanic acid appears to cause immunosuppression primarily via a $T$ cell dependent manner [23, 24]. It is possible that the trend for lower vitamin $\mathrm{D}$ and cis-UCA levels in MS patients are each indicative of lower UV exposure, rather than as mediators of disease themselves. 

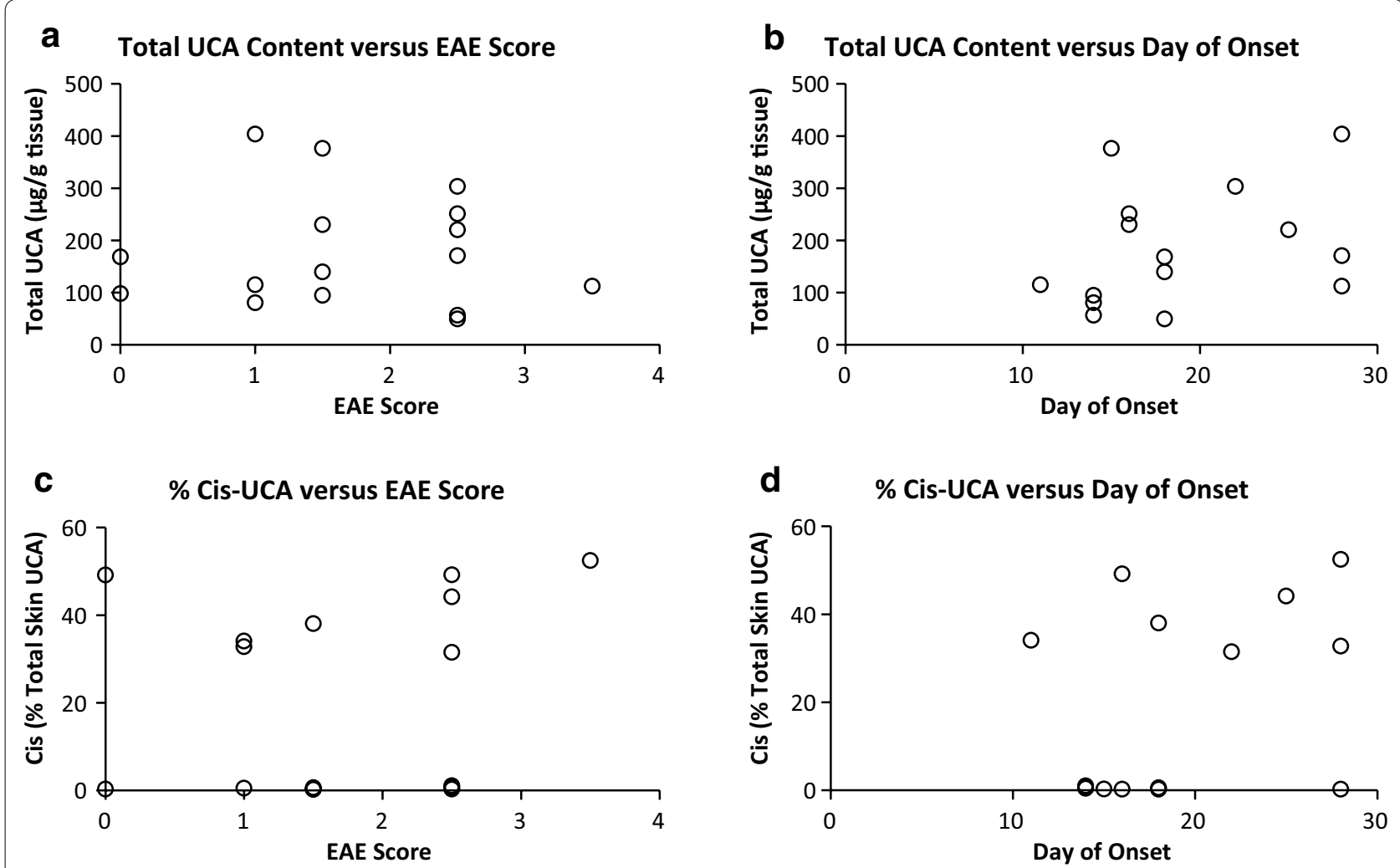

Fig. 5 UCA content does not correlate with EAE disease index. There is no correlation between total UCA content (a-b) nor \% UCA in the cis isomer $(\mathbf{c}-\mathbf{d})$, and terminal EAE score $(\mathbf{a}, \mathbf{c})$ or day of onset $(\mathbf{b}, \mathbf{d})$

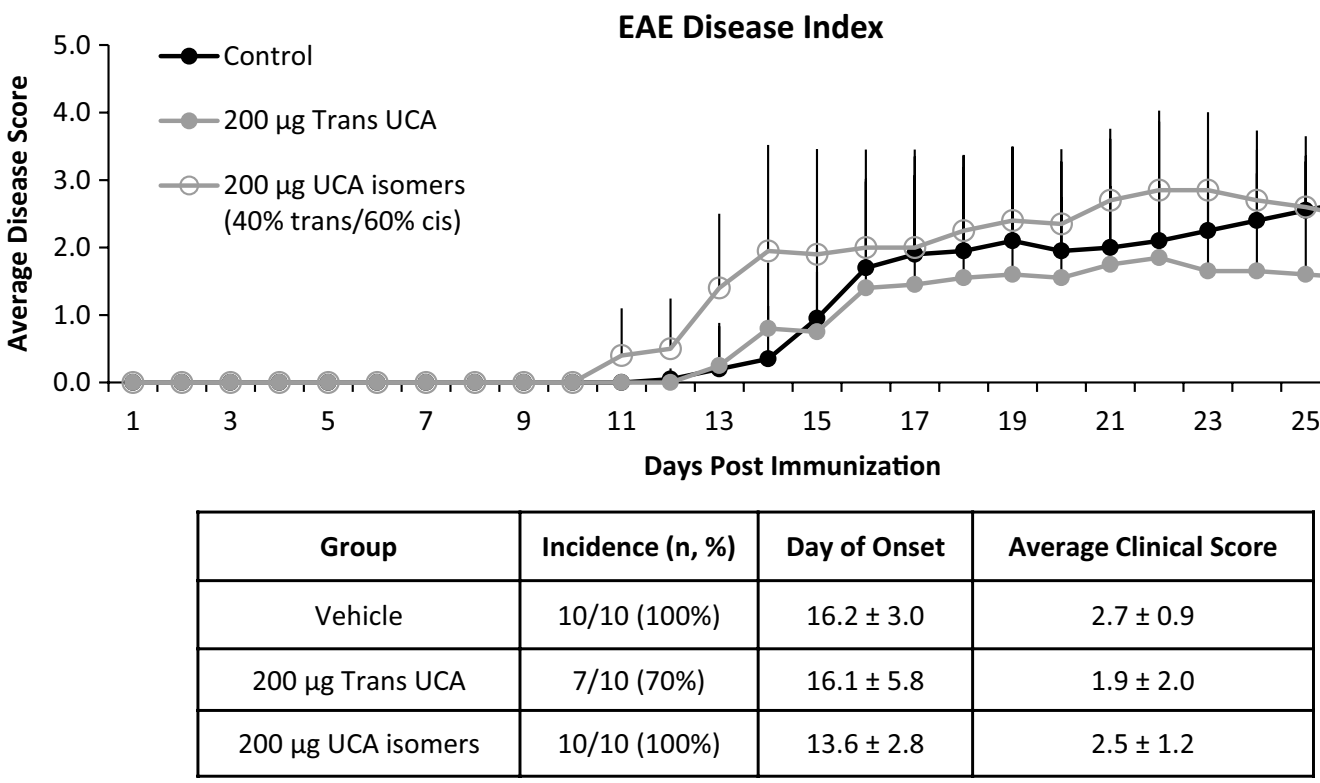

Fig. 6 Systemic delivery of UCA via i.p. injection does not prevent development of EAE. Neither day of onset nor disease progression is significantly altered by either trans- or mixed UCA isomers. Data are representative of one experiment 
Additional work is needed to explore avenues other than vitamin $\mathrm{D}$ and urocanic acid to determine by what mechanism UVB is providing protection against autoimmune disease.

\section{Abbreviations}

MS: Multiple sclerosis; 25-OH-D: 25-hydroxyvitamin D; EAE: experimental autoimmune encephalomyelitis; UV(B): ultraviolet (B); UCA: urocanic acid.

\section{Authors' contributions}

Contributed to conception and design: Al, SM, LP, HD contributed to acquisition of data: Al, SM contributed to analysis and editing of data: Al, SM drafted and/or revised the article: AI, SM, LP, HD all authors read and approved the final manuscript

\section{Acknowledgements}

We thank Ellen Lake for technical assistance and Pat Mings and Deb Noltner for their assistance in preparation of the manuscript.

\section{Competing interests}

The authors declare that they have no competing interests.

\section{Availability of data and material}

The data generated during the current study are available from the corresponding author on reasonable request.

\section{Ethics approval}

All animal procedures were approved by the Research Animal Resources Committee of the College of Agricultural and Life Sciences at the University of Wisconsin-Madison.

\section{Funding}

This work was supported by a fund from the Wisconsin Alumni Research Foundation.

Received: 3 August 2016 Accepted: 14 December 2016

Published online: 05 January 2017

\section{References}

1. Kampman MT, Steffensen LH, Mellgren SI, Jorgensen L. Effect of vitamin D3 supplementation on relapses, disease progression, and measures of function in persons with multiple sclerosis: exploratory outcomes from a double-blind randomised controlled trial. Mult Scler J. 2012;18:1144-51.

2. Stein M, Liu Y, Gray O, Baker J, Kolbe S, Ditchfield M, et al. A randomized trial of high-dose vitamin D2 in relapsing-remitting multiple sclerosis. Neurology [Internet]. 2011;77:1611-8. doi:10.1212/ WNL.0b013e3182343274.

3. Røsjø E, Steffensen LH, Jørgensen L, Lindstrøm JC, Šaltytè Benth J, Michelsen AE, et al. Vitamin D supplementation and systemic inflammation in relapsing-remitting multiple sclerosis. J Neurol. 2015;262:2713-21.

4. Meehan TF, Vanhooke J, Prahl J, DeLuca HF. Hypercalcemia produced by parathyroid hormone suppresses experimental autoimmune encephalomyelitis in female but not male mice. Arch Biochem Biophys. 2005:442:214-21.
5. Cantorna MT, Humpal-Winter J, DeLuca HF. Dietary calcium is a major factor in 1,25-dihydroxycholecalciferol suppression of experimental autoimmune encephalomyelitis in mice. J Nutr. 1999;129:1966-71.

6. Wang Y, Marling SJ, Zhu JG, Severson KS, DeLuca HF. Development of experimental autoimmune encephalomyelitis (EAE) in mice requires vitamin D and the vitamin D receptor. Proc Natl Acad Sci. 2012;109:8501-4.

7. Deluca HF, Plum LA. Vitamin D deficiency diminishes the severity and delays onset of experimental autoimmune encephalomyelitis. Arch Biochem Biophys. 2011;513:140-3.

8. Fisher MS, Kripke ML. Systemic alteration induced in mice by ultraviolet light irradiation and its relationship to ultraviolet carcinogenesis. Proc Natl Acad Sci. 1977;74:1688-92

9. Wang Y, Marling SJ, Mcknight SM, Danielson AL, Severson KS, Deluca HF. Suppression of experimental autoimmune encephalomyelitis by 300 $315 \mathrm{~nm}$ ultraviolet light. Arch Biochem Biophys [Internet]. 2013;536:81-6. doi:10.1016/j.abb.2013.05.010.

10. Baden HP, Pathak MA. The metabolism and function of urocanic acid in skin. J Invest Dermatol. 1967:48:11-7.

11. Gibbs NK, Norval M. Urocanic acid in the skin: A mixed blessing? J Invest Dermatol. 2011:131:14-7.

12. Gibbs NK, Norval M, Traynor NJ, Wolf M, Johnson BE, Crosby J. Action spectra for the trans to cis photoisomerisation of urocanic acid in vitro and in mouse skin. Photochem Photobiol. 1993;57:584-90.

13. Correale J, Farez MF. Modulation of multiple sclerosis by sunlight exposure: role of cis-urocanic acid. J Neuroimmunol. 2013;261:134-40.

14. Davis GE, Lowell WE. Indirect evidence that ultraviolet-B radiation mitigates multiple sclerosis in the United States. J Photochem Photobiol B Biol. 2015;151:46-7.

15. Spelman T, Gray O, Trojano M, Petersen T, Izquierdo G, Hupperts R, et al. Seasonal variation of relapse rate in multiple sclerosis is latitude dependent. Ann Neurol. 2014;76:880-90.

16. de Abreu DAF, Ibrahim EC, Boucraut J, Khrestchatisky M, Feron F. Severity of experimental autoimmune encephalomyelitis is unexpectedly reduced in mice born to vitamin D-deficient mothers. J Steroid Biochem Mol Biol. 2010;121:250-3.

17. El-Ghorr A, Norval M. The effect of chronic treatment of mice with urocanic acid isomers. Photochem Photobiol. 1997;65:866-72

18. Laurema A, Aioi A, Maibach H. Topical cis-urocanic acid suppresses both induction and elicitation of contact hypersensitivity in BALB/C mice. Acta Derm Venereol. 1995:75:272-5.

19. Kurimoto I, Streilein JW. cis-Urocanic acid suppression of contact hypersensitivity induction is mediated via tumor necrosis factor-alpha. J Immunol. 1992;148:3072-6.

20. Kammeyer A, Pavel S, Asghar SS, Bos JD, Teunissen MB. Prolonged increase of cis-urocanic acid levels in human skin and urine after single total-body ultraviolet exposures. Photochem Photobiol. 1997:65:593-8.

21. Anglin JJ, Bever A, Everett M, Lamb J. Ultraviolet-light-induced alterations in urocanic acid in vivo. Biochim Biophys Acta. 1961:53:408-9.

22. Kok LF, Marsh-Wakefield F, Marshall JE, Gillis C, Halliday GM, Byrne SN. B cells are required for sunlight protection of mice from a CNS-targeted autoimmune attack. J Autoimmun [Internet]. 2016;1. http://linkinghub. elsevier.com/retrieve/pii/S0896841116300774

23. Halliday GM, Damian DL, Rana S, Byrne SN. The suppressive effects of ultraviolet radiation on immunity in the skin and internal organs: implications for autoimmunity. J Dermatol Sci. 2012;66:176-82.

24. Yamaguchi T, Hiromasa K, Kabashima-Kubo R, Yoshioka M, Nakamura M. Galectin-7, induced by cis-urocanic acid and ultraviolet B irradiation, down-modulates cytokine production by T lymphocytes. Exp Dermatol. 2013;22:840-2. 